\title{
State Curves and Flipping for an Orbiting Cylinder at Low Reynolds Numbers
}

\section{Baranyi}

\begin{abstract}
Sudden changes found in the time-mean and rms values of force coefficients of a circular cylinder in forced orbital motion placed in a uniform stream when plotted against ellipticity of the orbital path suggest that two solutions (states) exist. This 2D numerical simulation was performed in order to gain further evidence of this hypothesis through flipping of the solution. Time histories and limit cycle curves of force coefficients for stationary, in-line, and orbital paths around the time of the flip were investigated, as well as time-mean and rms values of lift, drag, and base pressure coefficients versus ellipticity for the flipped solution. Results provide evidence of the existence of two solutions.
\end{abstract}

Keywords Orbiting cylinder - Numerical simulation · Flipping · 2D flow · Low Reynolds number

\section{Introduction}

Although there are countless studies for flow around a circular cylinder, either stationary or oscillating in one direction, investigations concentrating upon orbital motions are still rather uncommon (see e.g. $[10,11]$ ). Among these, studies of a cylinder in forced orbital motion in a uniform stream are relatively rare. Didier and Borges [7] were able to identify lock-in for in-line and transverse cylinder oscillation and for a cylinder orbiting in a circular path.

Lu and Dalton [8], working with forced transverse cylinder oscillation and investigating the effect of oscillation frequency, found switches in flow patterns and sudden $180^{\circ}$ phase angle change between lift and cylinder displacement. Blackburn and Henderson [6] confirmed these findings, as well as identifying sudden changes in energy transfer between cylinder and fluid.

\footnotetext{
L. Baranyi

Department of Fluid and Heat Engineering, University of Miskolc,

H-3515 Miskolc-Egyetemváros, Hungary

e-mail: arambl@uni-miskolc.hu
} 
In earlier studies, the author identified sudden jumps in the time-mean and rms values of force coefficients when plotted against ellipticity of orbit. Since these sudden jumps occur between two envelope curves [2], the author's hypothesis is that there are two solutions (states) that characterise the wake flow, and these represent changes in the structure of flow patterns. These jumps were investigated for several cases at different Reynolds numbers, and at different amplitudes of the in-line component of orbit [3]. A pre- and post-jump study also incorporated energy transfer, limit cycle curves, phase angle differences, and flow patterns [4]. All showed sudden switches, in agreement with the forced transverse results of [6] and [8].

This study attempts to gather further evidence of wake behaviour through flipping the solutions, as this has been recommended as one method of confirming the presence of two solutions [5].

\section{Governing Equations and Numerical Method}

The dimensionless governing equations for an incompressible constant property Newtonian fluid flow around an orbiting circular cylinder are the two components of the Navier-Stokes equations, the continuity equation and pressure Poisson equation written in a non-inertial system fixed to the cylinder:

$$
\begin{aligned}
& \frac{\partial u}{\partial t}+u \frac{\partial u}{\partial x}+v \frac{\partial u}{\partial y}=-\frac{\partial p}{\partial x}+\frac{1}{\operatorname{Re}} \nabla^{2} u-a_{0 x}, \\
& \frac{\partial v}{\partial t}+u \frac{\partial v}{\partial x}+v \frac{\partial v}{\partial y}=-\frac{\partial p}{\partial y}+\frac{1}{\operatorname{Re}} \nabla^{2} v-a_{0 y}, \\
& D=\frac{\partial u}{\partial x}+\frac{\partial v}{\partial y}=0, \\
& \nabla^{2} p=2\left[\frac{\partial u}{\partial x} \frac{\partial v}{\partial y}-\frac{\partial u}{\partial y} \frac{\partial v}{\partial x}\right]-\frac{\partial D}{\partial t}
\end{aligned}
$$

In these equations $\nabla^{2}$ is the 2D Laplacian operator, $x, y$ are Cartesian co-ordinates, $u, v$ are the $x, y$ components of velocity in the system fixed to the cylinder, $a_{0 x}, a_{0 y}$ are the components of cylinder acceleration, $p$ is the pressure, $D$ is dilation. Here $\operatorname{Re}$ is the Reynolds number, $\operatorname{Re}=U d / v$ where $d$ is the cylinder diameter, $U$ is the free stream velocity and $v$ is the kinematic viscosity.

Although in Equation (4) the dilation $D=0$ by continuity (3), I retain its partial derivative with respect to time to reduce numerical errors. Equations (1), (2) and (4) will be solved while the continuity equation (3) is satisfied at every time step.

No-slip boundary condition is used on the cylinder surface for the velocity and a Neumann-type condition is used for pressure $p$. A potential flow distribution is assumed far from the cylinder. 
Boundary fitted coordinates are used to impose the boundary conditions accurately. Using unique, single-valued functions, the physical domain bounded by two concentric circles can be mapped into a rectangular computational domain where the spacing is equidistant in both directions. In the physical domain logarithmically spaced radial cells are used, providing a fine grid scale near the cylinder wall and a coarse grid in the far field. Using the mapping functions, not specified here, the governing equations and boundary conditions are transformed into the computational plane. The transformed equations are solved by using the finite difference method. For further details see [1].

The 2D code developed by the author has been extensively tested against experimental and computational results for a stationary cylinder and good agreement has been found [1]. The code was extended first for an oscillating and then for an orbiting cylinder. For this study the dimensionless time step was 0.0005 and the number of grid points $301 \times 177$. For all Re investigated in this study $(\operatorname{Re}=120-180)$ the solution was grid independent. The ratio of the radius of the outer computational domain and cylinder radius was 40 .

Figure 1 shows the flow arrangement. The motion of the centre of the cylinder with unit diameter is specified as follows:

$$
x_{0}(t)=A_{x} \cos (2 \pi f t), \quad y_{0}(t)=-A_{y} \sin (2 \pi f t)
$$

where $f$ is the dimensionless oscillation frequency, $A_{x}, A_{y}$ are the dimensionless amplitudes of oscillations in $x$ and $y$ directions, respectively. In Fig. $1 U$ is the free stream velocity. Here the frequencies in the two directions are identical, which for nonzero $A_{x}, A_{y}$ amplitudes gives an ellipse, shown in the dotted line in the figure. If one of the amplitudes is zero, in-line or transverse oscillation is obtained. $A_{x}$ alone yields pure in-line oscillation, and then as $A_{y}$ is increased, the ellipticity $e=A_{y} / A_{x}$ increases to yield a full circle at $e=1$. The negative sign in $y_{0}$ in Equation (5) makes the cylinder orbit clockwise (clw); by changing this sign of $y_{0}$ an anticlockwise (aclw) orbit is obtained.

Fig. 1 Cylinder in orbital motion

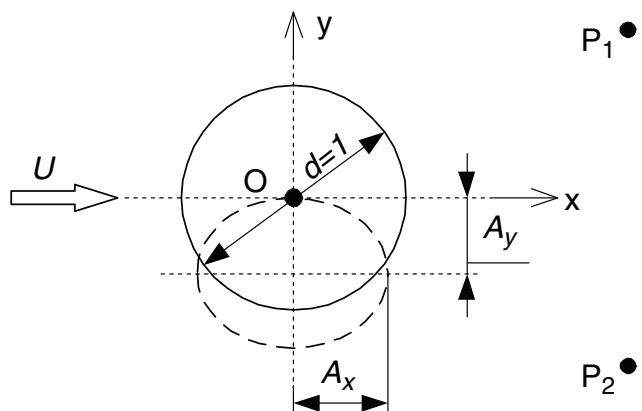




\section{Computational Results}

During each set of computations $\operatorname{Re}$ and $A_{x}$ are fixed and $f$ is kept constant at some percentage of Strouhal number $\mathrm{St}_{0}$ (the frequency of vortex shedding from a stationary cylinder at that Re). In this study this percentage was between 70-105 to ensure lock-in at moderate oscillation amplitudes.

An interesting phenomenon was observed when looking at the time-mean value (TMV) and root-mean-square ( $r m s$ ) values of lift, drag and base pressure coefficients for an orbiting cylinder in a uniform flow. Abrupt jumps were found when these values were plotted against ellipticity $e$ with $\mathrm{Re}$ and $A_{x}$ kept constant, [3]. A typical example for the TMV of lift coefficient for both clockwise and anticlockwise direction of orbit is shown in Fig. 2 a for Re $=160, A_{x}=0.4, f=0.85 \mathrm{St}_{0}$. The filled triangles show results for a cylinder orbiting anticlockwise (aclw in the figure). Note that there are two envelope curves, which are roughly parallel with each other and of negative slope. On the other hand, the empty squares in Fig. 2a show results for a clockwise (clw) orbit, with the other parameters unchanged. The two envelope curves can be seen, again roughly parallel, but the slope is positive, and they are a mirror image of the envelope curves of the cylinder orbiting anticlockwise. Although it cannot be seen well at small $e$ values in the figure, there are eight jumps or switches in state. For both directions of orbit the jumps occur at the same ellipticity values and computational points for the two cases lie on different state curves except for the values near $e=0$. In all calculations made so far, $C_{\text {Lmean }}$ has shown this pattern. Time histories of $C_{\text {Lmean }}$ before and after the jumps are substantially different, [2].

The TMV and rms of drag and base pressure, further the rms of lift, behaved differently from $C_{\text {Lmean }}$, characterised by two state curves which are not parallel but intersect each other at $e=0$. A typical example is shown in Fig. 2b. The main parameters $\left(\operatorname{Re}, A_{x}\right.$ and $f$ ) are the same as in Fig. 2a. From the sets of computations, it is clear that the two pairs of envelope or state curves are independent of the direction of orbit. Here the computational points belonging to the same $e$ values coincide with each other and thus naturally lie on the same envelope curve. This is reassuring in two ways: (1) The code produces the same time-mean and rms results for two
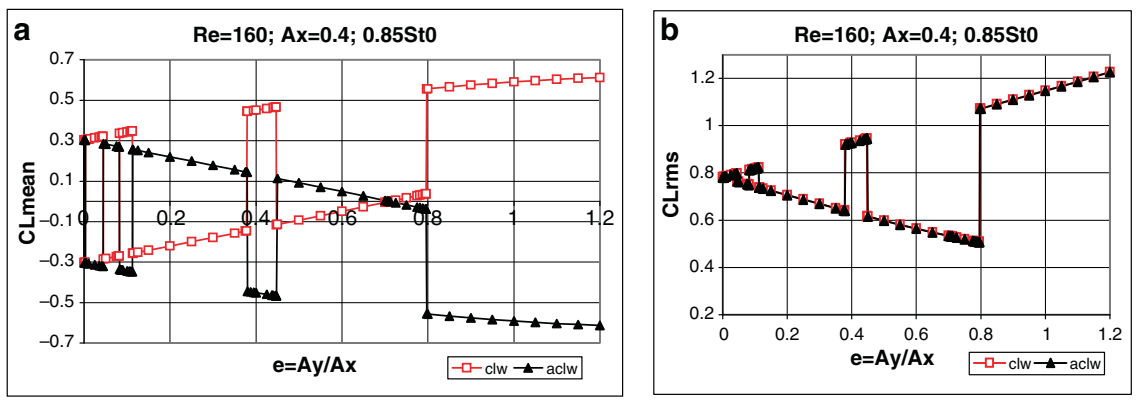

Fig. 2 Time-mean and $r m s$ values of lift versus ellipticity for clockwise (clw) and anticlockwise (aclw) direction of orbit $\left(\mathrm{Re}=160 ; A_{x}=0.4, f=0.85 \mathrm{St}_{0}=0.15997\right)$ 
different situations represented by the two directions of orbit, and this confirms that the code is consistent, and (2) the existence of envelope curves is proved by results obtained for two different cases. This finding also supports the idea that there are two states or solutions and the solution jumps from one state to the other and back.

To sum up the findings, there appear to be two states between which the solution switches which indicates a strong possibility of bifurcation. The two solutions can be obtained (a) by using different initial conditions or (b) by flipping the solution. It was shown in [4] that changing the initial condition for the orbiting cylinder yields two different solutions. With flipping, if both states are obtained, this is additional evidence for a two-state solution. For this purpose the solution for a circular cylinder (stationary, moving in-line, or in orbital motion) placed in an otherwise uniform flow is flipped. This is done by replacing every quantity (velocity and pressure fields) by its mirror image values, at one instant $(\Theta \rightarrow 2 \pi-\Theta)$ without changing the cylinder motion, where $\Theta$ is the polar angle. Time-histories and limit cycles were plotted in order to compare pre-flip and post-flip solutions. Two types of limit cycles were produced: one for two components of flow velocity at a point, and the other for drag and lift coefficients. To check that the code for flipping was effective, the least complicated cases were attempted first.

\subsection{Flipping for a Stationary Cylinder}

Computations were carried out for a stationary cylinder at $\mathrm{Re}=180 \mathrm{for}$ the dimensionless time interval of $[0,500]$ and the solution was flipped at $t_{1}=250$ when the flow was already periodic (limit cycle). Time histories of lift and drag coefficients and those of $u$ and $v$ velocity components were stored at points $\mathrm{P}_{1}(2,1)$ and $\mathrm{P}_{2}(2,-1)$, as these points have been shown to be reliable for experimental measurement of velocity signals (see [9]). Points $\mathrm{P}_{1}$ and $\mathrm{P}_{2}$, shown in Fig. 1, are mirror images of each other and are located in the wake of the cylinder on the physical plane, where the origin of the coordinate system is fixed to the centre of the cylinder and coordinates are made dimensionless by the cylinder diameter $d$. Time history and limit cycle curves for velocity components at these points, i.e. $\left(u_{1}, v_{1}\right),\left(u_{2}, v_{2}\right)$ and limit cycle curves for force coefficients $\left(C_{D}, C_{L}\right)$ were plotted before and after the flip. It was found that:

- All three limit cycle curves mentioned remain unchanged after flipping.

- There was an approximately $180^{\circ}$ phase shift in $C_{L}(t)$ at flipping and practically no phase shift in $C_{D}(t)$.

- For a stationary cylinder the shape of lift and drag coefficient signals are regular and this feature of the solution is preserved after the flipping as well.

- Due to symmetry in the position of points $\mathrm{P}_{1}$ and $\mathrm{P}_{2}$, limit cycle curves for the velocity components are mirror images of each other, i.e. $\left(u_{1}, v_{1}\right)=\left(u_{2},-v_{2}\right)$ and $\left(u_{2}, v_{2}\right)=\left(u_{1},-v_{1}\right)$.

All these expected results serve to show that the code works well. Due to lack of space no figures are included for the stationary cylinder. 


\subsection{Flipping for In-Line Oscillation}

Computations were carried out for a cylinder oscillating in-line at $\mathrm{Re}=180$ with $A_{x}=0.3$ amplitude and $f=0.9 \mathrm{St}_{0}=0.1737$ frequency. Flipping was carried out when the oscillating cylinder reached its furthest downstream position $\left(x_{0}=A_{x}\right)$. In this case, velocity signals were measured at points $\mathrm{P}_{1}$ and $\mathrm{P}_{2}$, which are fixed to the oscillating cylinder. In this way, the velocity components at the two points are relative velocities measured in the coordinate system fixed to the cylinder.

- Figure 3 shows the $C_{L}(t)$ signal around the flip $\left(t_{1}=247.5535\right)$. The shape of the signal, after a short transitional period, is reversed, i.e. the more rounded peaks switch from bottom to top. This is evidence for the existence of two solutions.

- Here, all limit cycle curves change with the flip. Still, some symmetries can be found between quantities before $\left(t<t_{1}\right)$ and after $\left(t>t_{1}\right)$ the flip, e.g.

$$
\begin{aligned}
& \left(u_{1}, v_{1} \mid t<t_{1}\right)=\left(u_{2},-v_{2} \mid t>t_{1}\right) \text { (see Fig. 4a, b) } \\
& \left(u_{2}, v_{2} \mid t<t_{1}\right)=\left(u_{1},-v_{1} \mid t>t_{1}\right) \text { (see Fig. 4c, d) } \\
& \left(C_{D}, C_{L} \mid t<t_{1}\right)=\left(C_{D},-C_{L} \mid t>t_{1}\right) \text { (see Fig. 5) }
\end{aligned}
$$

Figure 5 shows the relationship between $C_{L}$ and $C_{D}$ through the flip. The thin and thick closed curves show the $\left(C_{D}, C_{L}\right)$ limit cycle curves before and after the flip, respectively. Arrows show the orientation of the curves. The thin straight line represents the flip, when the solution jumps between the two states.

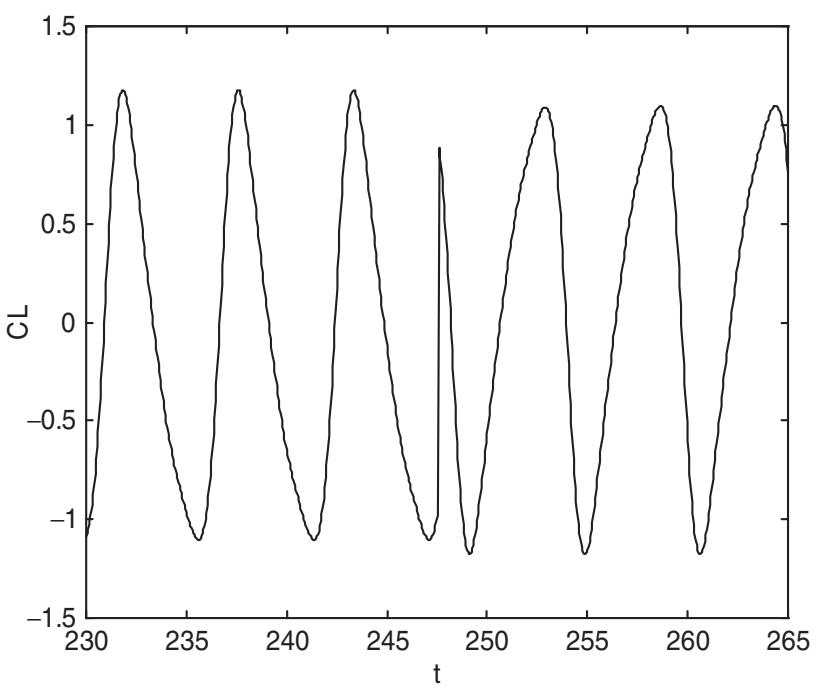

Fig. 3 Time history of lift coefficient in the vicinity of the flip 


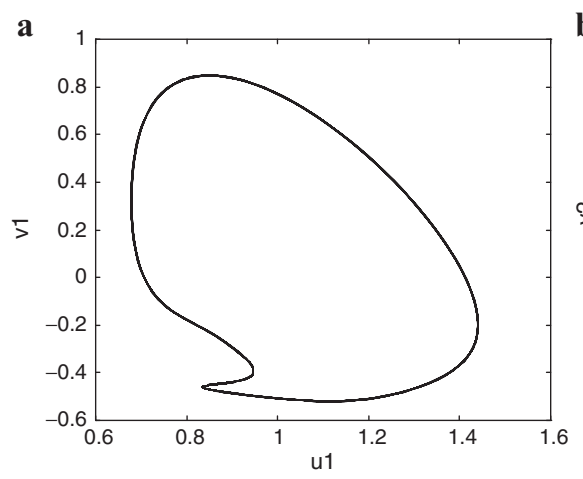

c

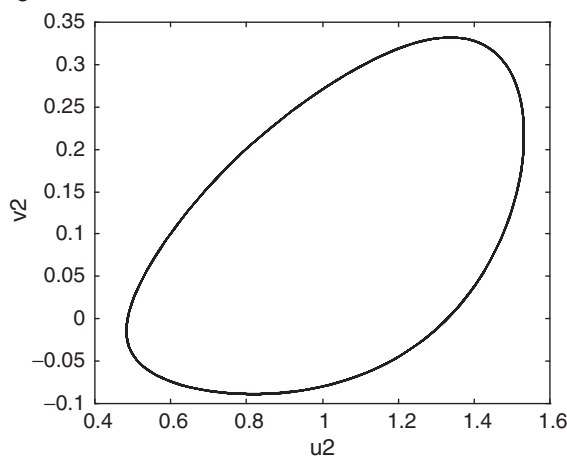

$\left(u_{2}, v_{2} \mid t<t_{1}\right)$

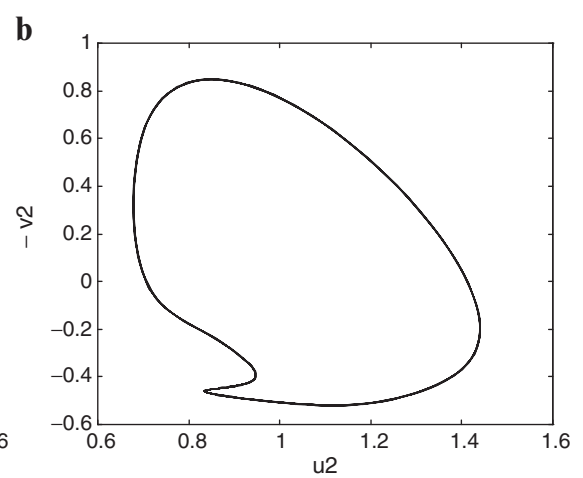

$\left(u_{2},-v_{2} \mid t>t_{1}\right)$

d

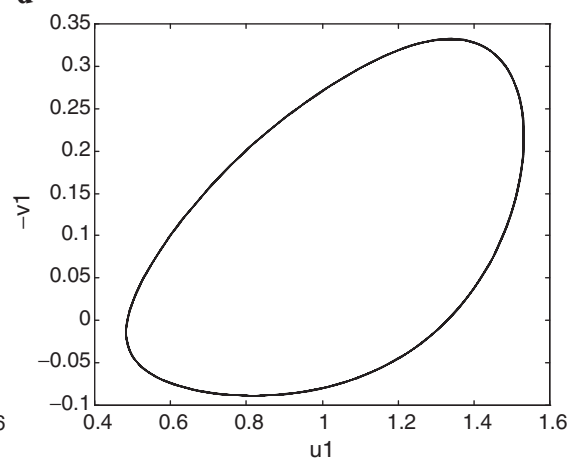

$\left(u_{1},-v_{1} \mid t>t_{1}\right)$

Fig. 4 Limit cycles for in-line cylinder oscillation $\left(\mathrm{Re}=180, A_{x}=0.3 ; f=0.1737 ; t_{1}=\right.$ 247.5535)

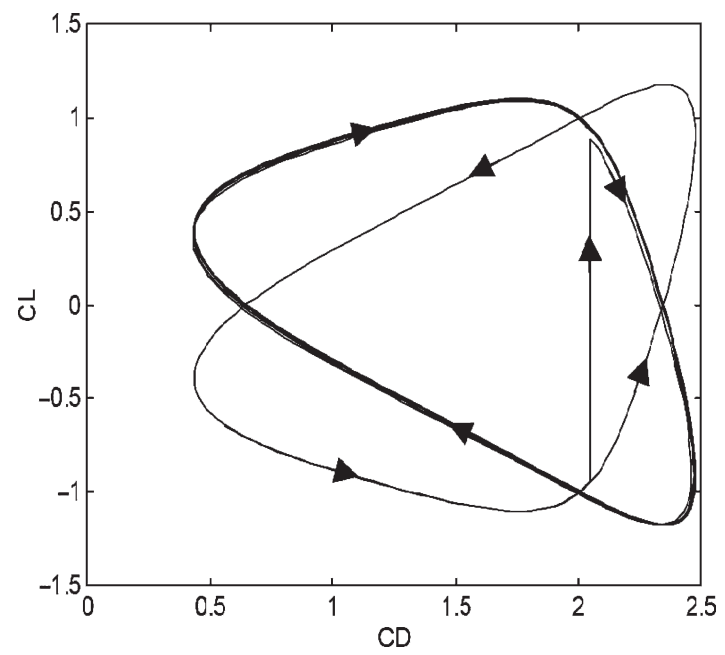

Fig. 5 Relationship between $C_{D}$ and $C_{L}$ near flip (parameters as in Fig. 4) 


\subsection{Flipping for Orbiting Cylinder}

To break the symmetry of in-line motion an orbiting cylinder was investigated, although due to lack of space we cannot go into detail here. A disturbance to in-line motion, the amplitude of transverse oscillation $A_{y}$, is chosen to be much smaller than $A_{x}$. In this way we expect that the symmetry features obtained for the cylinder oscillating in-line are not much distorted. The main parameters of the investigated case are: $\operatorname{Re}=160 ; A_{x}=0.3, A_{y}=0.012$ and $f=0.9 \mathrm{St}_{0}=0.16938, t_{1}=$ 247.5535 .

- The $C_{L}(t)$ signal around the flip is reversed, similarly to the one shown in Fig. 5 after a short transitional period; i.e. the more rounded peaks switch from bottom to top.

- Limit cycle curves show similar features, with the difference that relations mentioned for limit cycle curves in Section 3.2 are just approximately true.

- Limit cycle $\left(C_{L}, y_{0}\right)$ alters to a near-mirror image with the flip, while $\left(C_{D}, x_{0}\right)$ hardly alters at all.

\subsection{Effect of Flipping on Time-Mean and rms of Force Coefficients}

For all investigated cases the cylinder was orbiting in clockwise direction. Sets of computations were performed to investigate the effect of flipping on the TMV and rms values of different force coefficients. Out of the four sets investigated, two patterns have been identified. Representatives are shown in Figs. 6 and 7, where the $C_{L m e a n}$ curves are plotted against ellipticity $e$. The main feature of the first pattern (see Fig. 6) is that solutions flipped when the cylinder position is characterised by $x_{0} \approx x_{0 \max }$ (' 3 o'clock' position) roughly correspond to the solutions belonging to the anticlockwise direction of orbit (see also Fig. 2a), while keeping the other
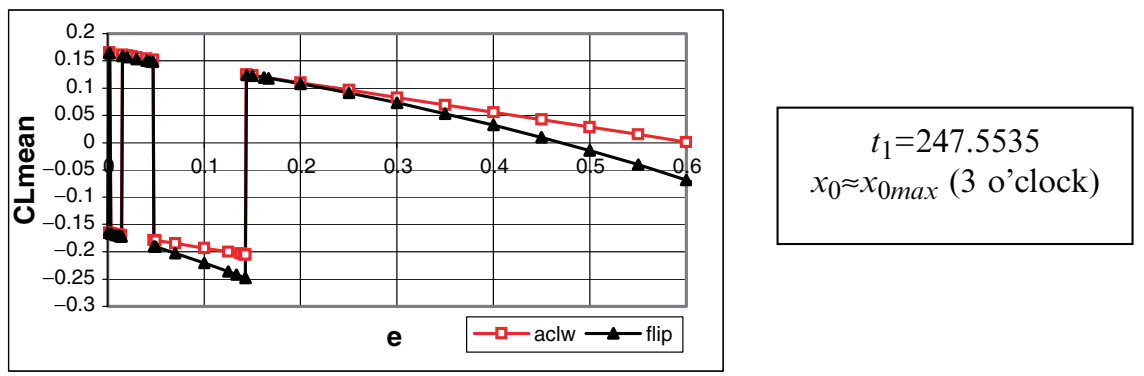

Fig. 6 Flipped and unflipped TMV of lift versus $e\left(\operatorname{Re}=160, A_{x}=0.3, f=0.9 \mathrm{St}_{0}=\right.$ $0.16938)$ 

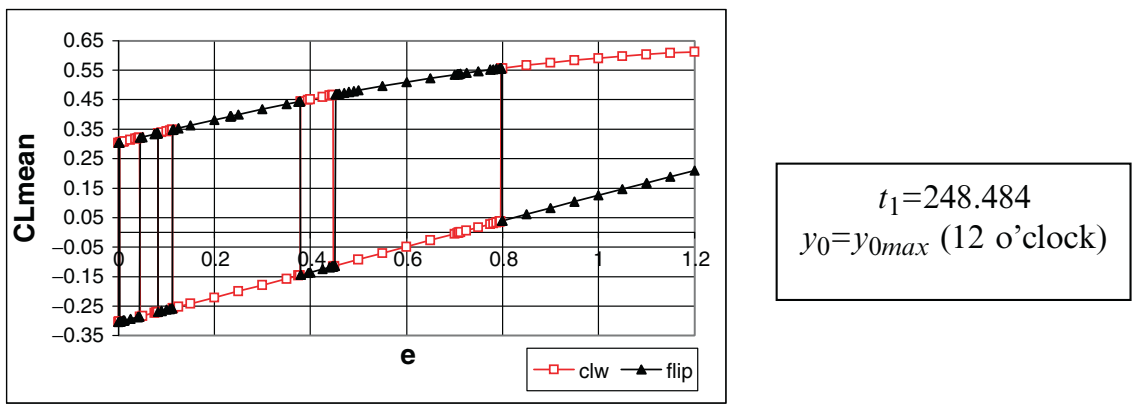

Fig. 7 Flipped and unflipped TMV of lift versus $e\left(\mathrm{Re}=160, A_{x}=0.4, f=0.85 \mathrm{St}_{0}=\right.$ $0.15997)$
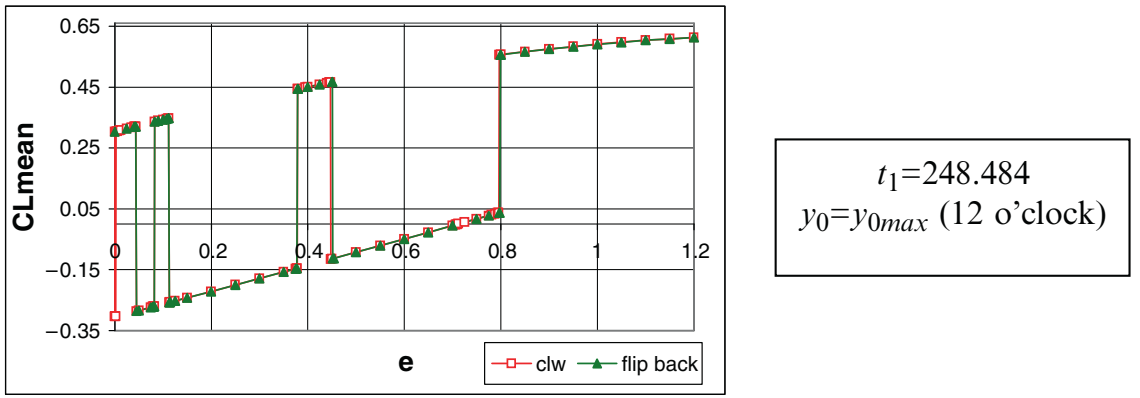

Fig. 8 Original and doubly flipped TMV of lift versus $e$ (as in Fig. 7)

parameters (Re, $A_{x}$ and $f$ ) unchanged. This means that $C_{\text {Lmean }}$ has negative slope, and that the flipped results are basically complementary to the values belonging to the case before flipping. The discrepancy tends to become larger with increasing $e$, but at times returns to near zero. If the solution is flipped once more (flipped back), however, we obtained an almost perfect reproduction of the original curve after two flippings! Although only $C_{\text {Lmean }}$ versus $e$ is shown here, similar results were obtained for the other TMV and $r m s$ values.

Figure 7 shows the other characteristic pattern found belonging to flipping time when the cylinder position is characterised by $y_{0}=y_{0 \max }$ (' 12 o'clock' position) Interestingly in this case the flipped solutions approximate the results belonging to the clockwise direction of orbit. The location of the jumps is unchanged and the flipped results are complementary to the unflipped solutions (i.e. can be found on the other state curve). In this respect the effect of flipping is very similar to that of changing the initial conditions for the cylinder motion [4]. The other surprising thing is that the flipped solution reproduces the state curves very accurately over the whole investigated $e$ domain. The flipped solution was flipped back; Figure 8 shows an almost perfect reproduction of the original curve after two flippings. At this stage it is unclear what kind of mechanism leads to either pattern 1 or pattern 
2. Hence further investigations are needed. It seems, though, that the position of the cylinder at the time when the flipping takes place has a crucial effect. Results for both patterns, however, give some extra evidence for the existence of a double solution, which seems as if it might be a case of bifurcation.

\section{Conclusions}

The effect of flipping on flow features for a stationary, oscillating or orbiting cylinder in a uniform stream was studied. Limit cycle and time history curves were investigated, and results for a cylinder either stationary or in in-line motion gave evidence for the existence of two solutions (states). Simulations of an orbiting cylinder with small cross-wise amplitude further supported this conclusion. The time-mean and rms values of force coefficients were investigated versus ellipticity $e$ for a cylinder orbiting clockwise, and two patterns were identified: (1) the flipped solution approximates the solution belonging to the anticlockwise orbit, discrepancy increasing with $e$, or (2) the flipped solution gave a very accurate complementary solution to the clockwise orbit, even when double-flipped.

Further research is needed to explain why two patterns appear, and to further clarify the phenomenon causing sudden changes in time-mean and rms values of force coefficients. POD analysis is planned to identify the type of bifurcation.

Acknowledgements The support provided by the Hungarian Research Foundation (OTKA, Project No. T 042961) is gratefully acknowledged. The author also thanks Prof. D. Barkley of Warwick University for his valuable advice, and Mr. S. Ujvárosi for his help in figure preparation.

\section{References}

1. Baranyi, L., Computation of unsteady momentum and heat transfer from a fixed circular cylinder in laminar flow. Journal of Computational and Applied Mechanics 4(1) (2003) 13-25.

2. Baranyi, L., Numerical simulation of flow past a cylinder in orbital motion. Journal of Computational and Applied Mechanics 5(2) (2004) 209-222.

3. Baranyi, L., Sudden jumps in time-mean values of lift coefficient for a circular cylinder in orbital motion in a uniform flow. In: 8th International Conference on Flow-Induced Vibration, Eds. E. de Langre and F. Axisa, Paris, II (2004) 93-98.

4. Baranyi, L., Energy transfer between an orbiting cylinder and moving fluid. In: Proceedings of the ASME Pressure Vessels and Piping Conference 9 (2007) 829-838.

5. Barkley, D., Private Communication, University of Warwick, December, 2005.

6. Blackburn, H.M., Henderson. R.D., A study of two-dimensional flow past an oscillating cylinder. Journal of Fluid Mechanics 385 (1999) 255-286.

7. Didier, E. Borges, A.R.J., Numerical predictions of low Reynolds number flow over an oscillating circular cylinder. In: Conference on Modelling Fluid Flow, Eds. T. Lajos and J. Vad, Budapest (2006) 165-172.

8. Lu, X.Y., Dalton, C., Calculation of the timing of vortex formation from an oscillating cylinder. Journal of Fluids and Structures 10 (1996) 527-541. 
9. Koide, M., Kubo, Y., Takahashi, T., Baranyi, L., Shirakashi, M., The vibration response of a cantilevered rectangular cylinder in cross-flow oscillation. Journal of Fluids Engineering 126, Trans. ASME (2004) 884-887.

10. Stansby, P.K., Rainey, R.C.T., On the orbital response of a rotating cylinder in a current. Journal of Fluid Mechanics 439 (2001) 87-108.

11. Williamson, C.H.K., Hess, P., Peter, M., Govardhan, R., Fluid loading and vortex dynamics for a body in elliptic orbits. In: Conference on Bluff Body Wakes and Vortex-Induced Vibration, Eds. P.W. Bearman and C.H.K. Williamson, Washington, DC (1998) 1-8. 